\title{
Noise and Equivalent Circuit of Double Injection
}

\author{
H. R. BILGER, * D. H. LEE, AND M-A. NICOLET \\ California Instilute of Technology, Pasadena, California 91109 \\ AND \\ E. R. MCCARTER \\ Esso Production Research Company, Houston, Texas 77001
}

(Received 5 August 1968)

\begin{abstract}
Measurements of the high-frequency noise of a silicon double-injection diode result in $\left\langle i^{2}\right\rangle=\alpha \cdot 4 k T(1 / r) \Delta f$ with $\alpha=1.04$ and in agreement with the literature. A new interpretation demands Nyquist noise with $\alpha \equiv 1$ in these devices at high frequencies. This is in accord with an equivalent circuit derived for the doubleinjection process. Speculations are made on the general validity of Nyquist noise in nonlinear devices at high frequencies. In addition, generation-recombination noise is suggested as the prime source of the lowfrequency noise.
\end{abstract}

\section{INTRODUCTION}

The literature reports on three separate experimental studies directly related to the present subject of highfrequency noise in double injection. ${ }^{1-8}$ Two of them report that the mean square of the current fluctuations can be represented by

$$
\left\langle i^{2}(\omega)\right\rangle=\alpha \cdot 4 k T g(\omega) \Delta f
$$

with $\alpha \sim 1$, although the two devices and their operating conditions are dissimilar. In one case, the structure is a long germanium diode operated in a square-law range. ${ }^{2}$ In the other, the device is a thin commercial germanium photocell operated in a $V^{3}$ range. ${ }^{3}$ Reference 1 relates to a long silicon diode operated in a square law range and the value of the limiting white-noise level is stated in terms of the low-frequency conductance $\partial I / \partial V$

$$
\left\langle i^{2}\right\rangle=\beta \cdot 4 k T(\partial I / \partial V) \Delta f
$$

with $\beta=0.52 \pm 0.1$, but the value of $g(\omega)$ at the measuring frequency is not given. The results of the present study (see Sec. II) establish that this value is $g(\omega)=$ $\frac{1}{2}(\partial I / \partial V)$. Thus, Eq. (1) also holds in this instance with $\alpha=1.0 \pm 0.2$.

This simple result obtained on dissimilar devices under different operating conditions seems to indicate a general fact, namely, that double injection exhibits thermal noise at high frequencies. Unfortunately, the mode of operation of the three devices used so far has been identified mainly through their $I-V$ characteristics. On the other hand, Rose shows in his "comparative anatomy of models for double injection" that the behavior of this type of current flow varies broadly at $\mathrm{dc}$ and depends on the relative values of such parameters as size, doping, mobilities, lifetimes, contacts, and transit time. ${ }^{4}$ In addition, double injection covers a

\footnotetext{
* Permanent address: Oklahoma State University, Stillwater, Okla.

M-A. Nicolet, H. R. Bilger, and E. R. McCarter, Appl. Phys. Letters 9, 434 (1966).

${ }^{2}$ S. T. Liu, S. Yamamoto, and A. van der Ziel, Appl. Phys. Letters 10, 308 (1967)

${ }^{3}$ F. Driedonks, R. J. J. Zijlstra, and C. Th. J. Alkemade, Appl. Phys. Letters 11, 318 (1967).

A. Rose, J. Appl. Phys. 35, 2664 (1964).
}

range of possible $I-V$ dependences which includes that of single injection. ${ }^{5}$ The $I-V$ characteristic, as such, is therefore an unreliable means with which to establish the presence of double injection, particularly if the physical parameters of the structure are not available for numerical checks. To clarify the situation, roomtemperature measurements are reported here on the noise and conductance of a diode whose behavior is first shown to agree closely with the predictions of the models developed for double injection.

Correspondingly, Sec. II derives an equivalent circuit from a physical model of double injection. Experimental results are obtained on a long silicon dode operated in the semiconductor regime $\left(I \propto V^{2}\right)$ which substantiate the model. Section III reports on noise measurements performed on the same device. An interpretation of the results and a general model of high-frequency noise is given in Sec. IV. Section V speculates on the origin of the excess noise observed in this diode.

\section{DIFFERENTIAL RESPONSE OF DOUBLE INJECTION}

Baron et al. ${ }^{6}$ point out that additional information on the properties of a double-injection diode can easily be obtained from its differential step response. Consider the case of an $I-V$ characteristic as shown in Fig. 1 , where $I \propto V^{2}$. If the current is indeed entirely due to double injection, a differential voltage step $\Delta V$ applied at $t=0$ should generate the current response shown by the solid line of Fig. 2. There is an instantaneous increase of current $\Delta i(0)$ at $t=0^{+}$followed by a second increase of $\Delta i(\infty)=\Delta i(0)$ after several time constants $\tau_{1}$. In general, the exponential part of the response will have an asymptotic amplitude $\Delta i(\infty)=$ $(n-1) \Delta i(0)$ if the dc characteristic is $I \propto V^{n}$. That this criterion is indeed satisfied in good double-injection diodes is demonstrated by the experimental result also recorded in Fig. 2. It was obtained on the sample of

${ }^{5}$ D. H. Lee and M-A. Nicolet, Solid-State Electron. 8, 182 (1965)

B. R. Baron, O. J. Marsh, and J. W. Mayer, J. Appl. Phys. 37, 


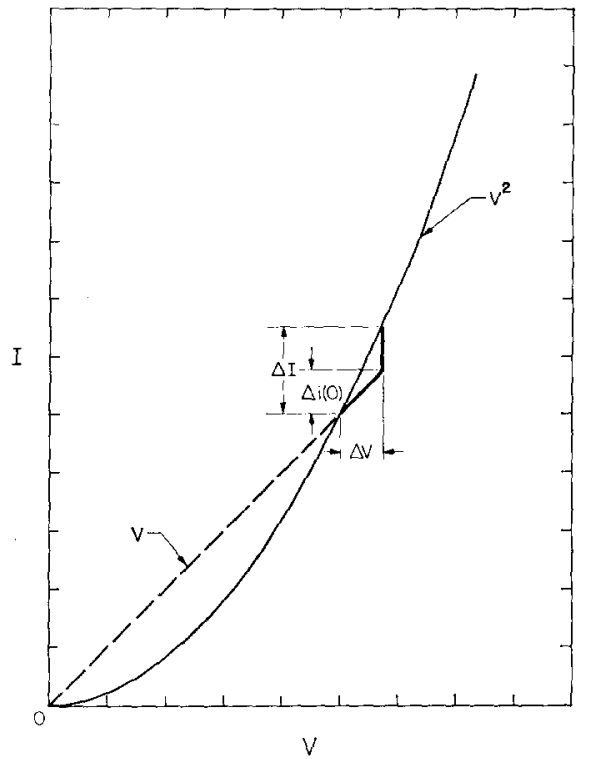

FIG. 1. Ideal characteristic of a double injection diode in the semiconductor regime $\left(I \propto V^{2}\right)$. The details are explained in Sec. II.

Fig. 3 at an operating point of $30 \mathrm{~V}$ and $1.6 \mathrm{~mA}$. The solid line is a least-squares fit to the experimental data.

The explanation of this behavior rests on the hypothesis that injected charge can not change instantaneously. Immediately after the application of the pulse $\Delta V$, the diode therefore behaves as a series of resistive elements whose values depend on the amount of charge stored in every interval $d x$ along the length $L$ of the diode. At $t=0^{+}$the resistance is therefore

$$
\begin{aligned}
r & =\int_{0}^{L}\left\{A q\left[\mu_{n} n(t=0)+\mu_{p} p(t=0)\right]\right\}^{-1} d x \\
& =\int_{0}^{L}[A \sigma(t=0)]^{-1} d x,
\end{aligned}
$$

where the symbols have their conventional meaning.

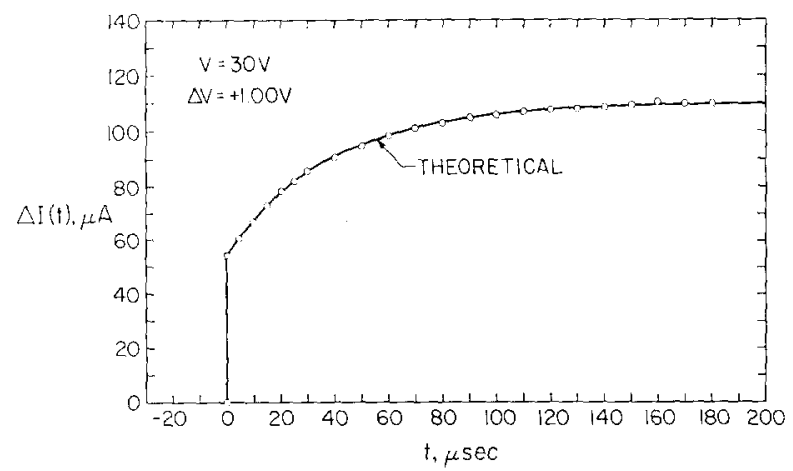

FIG, 2. Current response of the diode of Fig. 3 to a differential voltage step of $+1 \mathrm{~V}$ at an operating point of $30 \mathrm{~V}, 1.6 \mathrm{~mA}$. The solid line is a least-squares fit of

$$
\Delta i(t)=\Delta i(0)+\Delta i(\infty)\left[1-\exp \left(t / \tau_{1}\right)\right]
$$

to the data. The values of the parameters are given in Table $I$.
Since, in general, the densities $n$ and $p$ of the electrons and holes vary with the distance $x$ the requirement that the current $\Delta i(0)$ be free of divergence demands that the additional field $\Delta E(x)$ generated by $\Delta V$ will not be constant either. It will, in fact, have to adjust itself everywhere such that $\Delta E(x) \cdot A \sigma(t=0)=\Delta i(0)$ is constant. This is achieved by minute rearrangements of charges in the bulk and within a time span of the order of the dielectric relaxation time $\theta=\epsilon / \sigma$, which is typically below the resolution of electronic equipment. Hence, $\Delta E(x) \cdot A \sigma(t=0)=\Delta i(0)$ and

$$
\int_{0}^{L} \Delta E(x) d x=\Delta V
$$

As long as diffusion contributes negligibly to the $\mathrm{dc}$

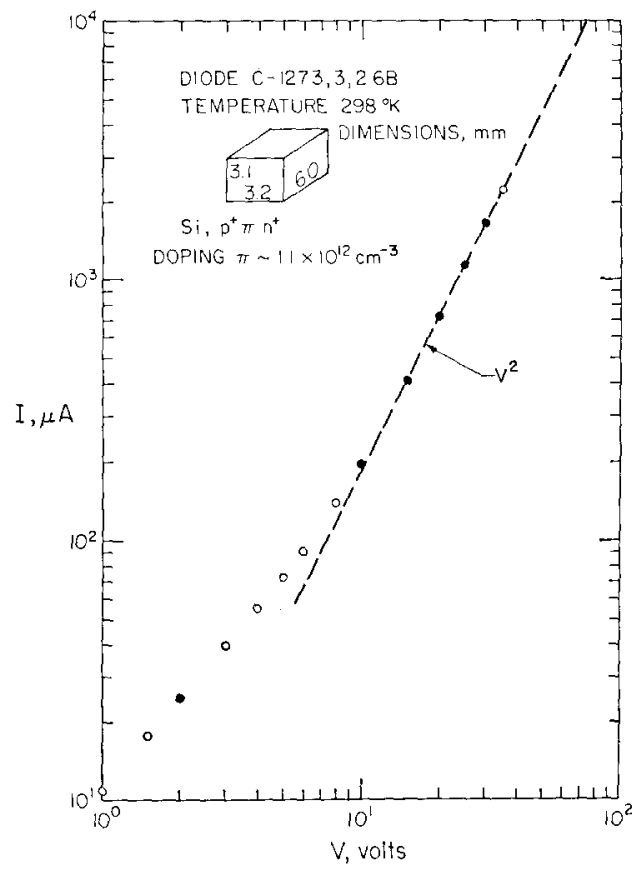

FIG. 3. I-V characteristic of the silicon double-injection diode studied at room temperature in this paper. Noise measurements are taken at $2,10,15,20,25$, and $30 \mathrm{~V}$ (see Sec. III).

current, the dc value $E(x)$ of the electric field satisfies $E(x) \cdot A \sigma(t=0)=I$, and

$$
\int_{0}^{L} E(x) d x=V
$$

It thus follows that

$$
1 / r=\Delta i(0) / \Delta V=I / V,
$$

as indicated in Fig. 1. At $t=\infty$, on the other hand,

$$
\Delta I / \Delta V=n(I / V) \text {. }
$$

Equations (4) and (5) hence state that the differential current will increase by $(n-1) \Delta i(0)$ between $t=0^{+}$ and $t=\infty$. If one further assumes the presence of a single linear recombination mechanism, this transition 
will take place with a single time constant $\tau_{1}$ and leads to a functional time dependence as shown in Fig 2.

The step response of a single-injection (unipolar space-charge-limited) current is quite different because the characteristic time scale is the transit time (of the order of $\left.L^{2} / \mu V\right)$, rather than a lifetime. ${ }^{7}$ If the current is a mixture of single and double injection or double injection with a significant contribution of diffusion, the clear identification of the mode of current flow becomes more difficult. To avoid such difficulties, the device of Figs. 2 and 3 has been selected. This structure is a duplicate of the diode studied in Ref. 1 and by Mayer $e t a l .{ }^{8}$ where a detailed analysis shows that the device conforms closely with the theoretical models developed by Baron. ${ }^{9}$

A linear equivalent circuit with one inductance and two resistances is shown in Fig. 4. The elements can easily be interpreted in terms of the physical mechanisms of the device. The parallel capacitance has been added to acount for the unavoidable electric field

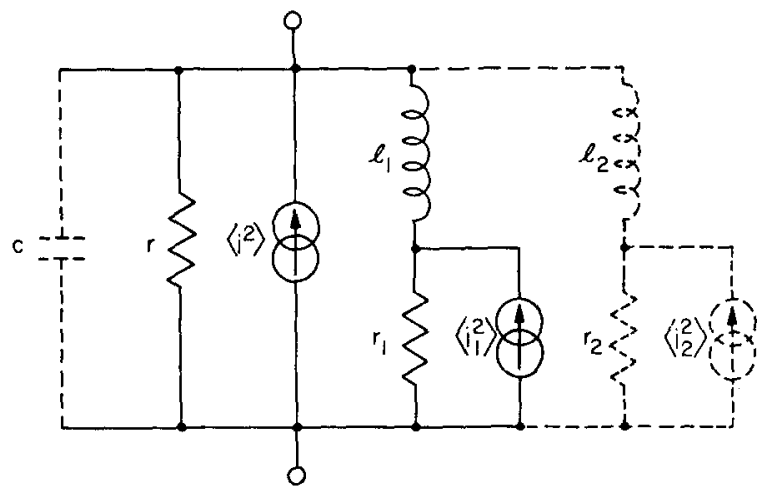

FIG. 4. Equivalent circuit. $\left\langle i^{2}\right\rangle=4 k T(1 / r) \Delta f$. The physical interpretation of the other elements is given in Secs. II, IV, and V.

across the terminals. The meaning of the resistance $r$ is discussed above. The branch of $l_{1}$ and $r_{1}$ represents the recombination mechanism responsible for the exponential part of the response, where $\tau_{1}=l_{1} / r_{1}$. In the presence of several competing recombination processes: this network will generally have to be complemented by a number of such $l-r$ branches connected in parallel as indicated by the dotted elements in Fig. 4, where each branch represents an independent recombination mechanism. For the present diode, the assumption of a single mechanism is sufficient, as demonstrated by Fig. 2.10

To test the validity of this equivalent circuit independently of the differential step response, the real and imaginary parts of the diode admittance have

${ }^{7}$ R. Baron, M-A. Nicolet, and V. Rodriguez, J. Appl. Phys. 37, 4156 (1966).

${ }_{8}^{8}$ J. W. Mayer, O. J. Marsh, and R. Baron, J. Appl. Phys. 39, 1447 (1968).

${ }^{9}$ R. Baron, J. Appl. Phys. 39, 1435 (1968).

${ }^{10} \mathrm{~A}$ careful experimental analysis revealed traces of a second time constant $\tau_{2}$ of about $\frac{1}{4} \tau_{1}$, but the effect was judged to be sufficiently small to be neglected.

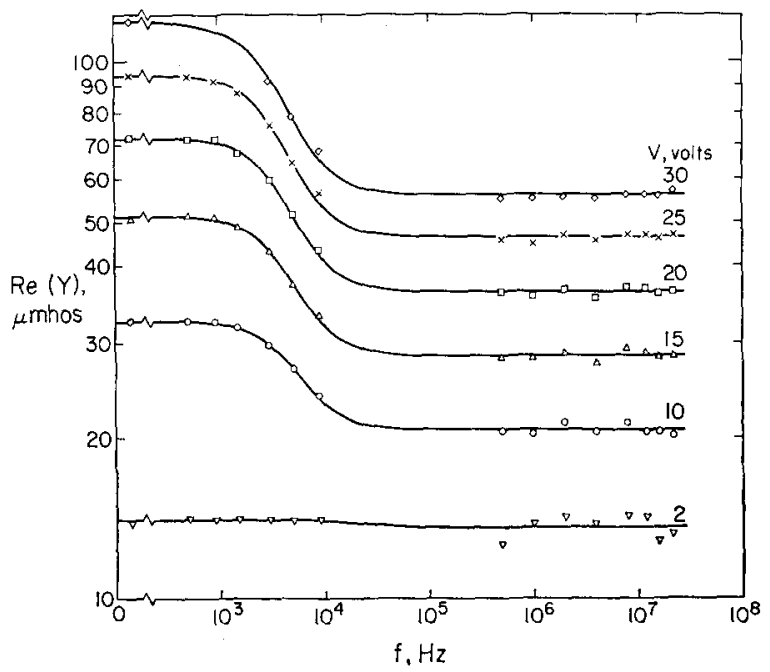

FIG. 5. Real part of the diode admittance vs frequency. The solid lines are least-squares fits to the data. The fitting function is that derived from the equivalent circuit of Fig. 4. The parameters obtained are given in Table I.

been measured from $90 \mathrm{~Hz}$ to $22 \mathrm{MHz}$. Some of the results are shown in Figs. 5 and 6. Also given there are the dependences predicted from the equivalent circuit of Fig. 4, using a least squares fit. The agreement is quite good. In Table I the values of $r, l_{1}$, and $r_{1}$ determined from the fits are compared with those obtained from the differential step response at various operating points. The discrepancies nowhere exceed $10 \%$. Above $20 \mathrm{~V}$ the ratios $l_{1} / r_{1}$ for the lifetime $\tau_{1}$ all lie between 35 and $41 \mu \mathrm{sec}$. This simple equivalent circuit thus adequately represents the small signal behavior of this double-injection diode from low frequencies up to over three decades above the transition point $\omega \tau_{1}=1$.

\section{HIGH-FREQUENCY NOISE OF DOUBLE INJECTION}

The previous section establishes that the silicon diode under investigation concurs with the predictions of the model for double injection above approximately

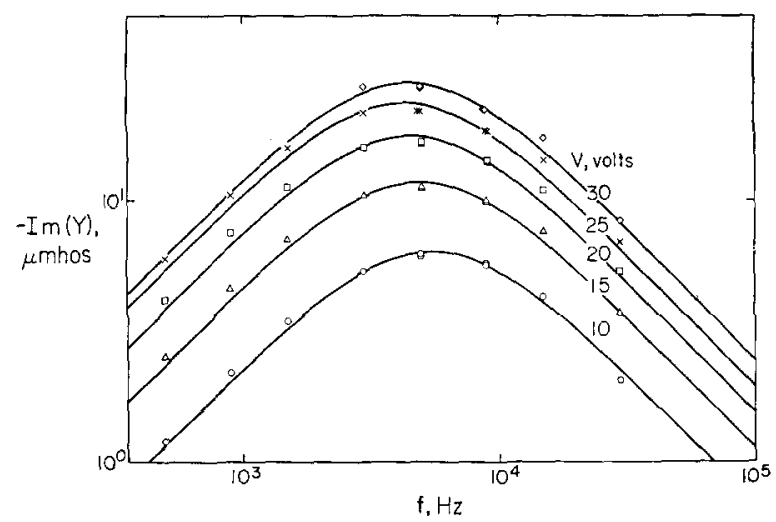

FIG. 6. Imaginary part of the diode admittance vs frequency. The solid lines are least-squares fits to the data. The fitting function is that derived from the equivalent circuit of Fig. 4 after subtraction of $\omega C=\operatorname{Im}(Y)_{f \rightarrow \infty}$. 
TABLE I. Values of the parameters for the equivalent circuit of Fig. 4 for the double-injection diode of Fig. 3.

\begin{tabular}{|c|c|c|c|c|c|c|c|c|c|}
\hline \multirow{2}{*}{\multicolumn{2}{|c|}{ Operating point }} & \multirow{2}{*}{\multicolumn{3}{|c|}{ From step response }} & \multicolumn{5}{|c|}{ From frequency response } \\
\hline & & & & & \multicolumn{3}{|c|}{$\operatorname{Re}(Y)$} & \multicolumn{2}{|c|}{$\operatorname{Im}(Y)$} \\
\hline$V(V)$ & $I(\mathrm{~mA})$ & $r(\mathrm{k} \Omega)$ & $l_{1}(H)$ & $r_{1}(\mathrm{k} \Omega)$ & $r(\mathrm{k} \Omega)$ & $l_{1}(\mathrm{H})$ & $r_{1}(\mathrm{k} \Omega)$ & $h(H)$ & $r_{1}(\mathrm{k} \Omega)$ \\
\hline 2.0 & 0.024 & 68 & $\cdots$ & $\cdots$ & 73.5 & $\cdots$ & $\cdots$ & $\cdots$ & $\cdots$ \\
\hline 10.0 & 0.196 & 47.1 & 2.4 & 81.9 & 48.7 & 2.6 & 81.4 & 2.3 & 78 \\
\hline 15.0 & 0.405 & 34.5 & 1.54 & 43.5 & 35.4 & 1.52 & 43.5 & 1.39 & 42.5 \\
\hline 20.0 & 0.710 & 26.7 & 1.08 & 29.7 & 27.0 & 1.08 & 28.7 & 1.01 & 28.5 \\
\hline 25.0 & 1.12 & 21.2 & 0.83 & 22.5 & 21.4 & 0.86 & 21.2 & 0.80 & 21.4 \\
\hline 30.0 & 1.60 & 18.1 & 0.70 & 18.5 & 17.8 & 0.69 & 16.8 & 0.63 & 17.9 \\
\hline
\end{tabular}

$10 \mathrm{~V}$. The diode therefore provides a sound basis for the noise measurements below.

The mean square of the current fluctuations $\left\langle i^{2}\right\rangle$ has been measured at six operating points from $60 \mathrm{kHz}$ to $22 \mathrm{MHz}$. Figure 7 shows the spectra in terms of the equivalent noise current $I_{\text {eq }}=\left\langle i^{2}\right\rangle / 2 q \Delta f$. It is seen that at all operating points the noise spectra reach a constant level above a limiting frequency which depends on the amount of excess noise present. The solid lines have been obtained by the least squares fitting of an assumed dependence $I_{\mathrm{eq}}=c_{1}+c_{2} / f^{c_{3}}$ to the data. The equivalent noise resistances $r_{\mathrm{eq}}=2 k T / q c_{1}$ at each operating point are listed in Table II.

The very close agreement between $r_{\mathrm{eq}}$ and the real part $r$ of the admittance at high frequencies indicates that

$$
\left\langle i^{2}\right\rangle=\alpha \cdot 4 k T g \Delta f
$$

with $\alpha \sim 1$ and $g=1 / r$. A better estimate of the value of $\alpha$ is derived in Fig. 8 where the high frequency noise levels are plotted against $g$. A least-squares fit yields

$$
\alpha=1.04
$$

with an estimated error of $\pm 5 \%$. It should be noted that this relationship holds at every operating point even where the square law dependence of the dc current does not hold. This observation is consistent with the results of Refs. 2 and 3. There, $\alpha \sim 1$ also holds, in

TABLE II. Comparison of the real part $r$ of the diode admittance

\begin{tabular}{|c|c|c|c|}
\hline \multicolumn{2}{|c|}{ Operating point } & \multirow{2}{*}{$\left({ }^{r} \Omega\right)$} & \multirow{2}{*}{$\left.\underset{(\mathrm{k} \Omega}{r_{\text {eq }}}\right)$} \\
\hline$V(\mathrm{~V})$ & $I(\mathrm{~mA})$ & & \\
\hline 2.0 & 0.024 & 73.5 & 70.5 \\
\hline 10.0 & 0.196 & 48.7 & 45.1 \\
\hline 15.0 & 0.405 & 35.4 & 33.4 \\
\hline 20.0 & 0.710 & 27.0 & 26.2 \\
\hline 25.0 & 1.12 & 21.4 & 21.4 \\
\hline 30.0 & 1.60 & 17.8 & 17.0 \\
\hline
\end{tabular}
with the equivalent noise resistance $r_{e q}$ at high frequencies. spite of the fact that the presence of pure double injection is questionable since Eq. (4) and (5) are not satisfied.

\section{MODEL OF HIGH-FREQUENCY NOISE FOR NONLINEAR DEVICES}

We propose a simple hypothesis to explain the results of Secs. II and III and of Refs. 1-3, namely,

$$
\alpha \equiv 1 \quad \text { at } \omega \tau_{1} \gg 1
$$

that is, such devices exhibit nothing but Nyquist noise of the resistance at high frequencies. ${ }^{11}$ In the device of this paper, the high-frequency resistance is $r$ (see Fig. 4), since the $l_{1}-r_{1}$ branch [has a large time constant $\tau_{1}$ equal to the lifetime of the carriers. This means, physically, that the recombination processes,

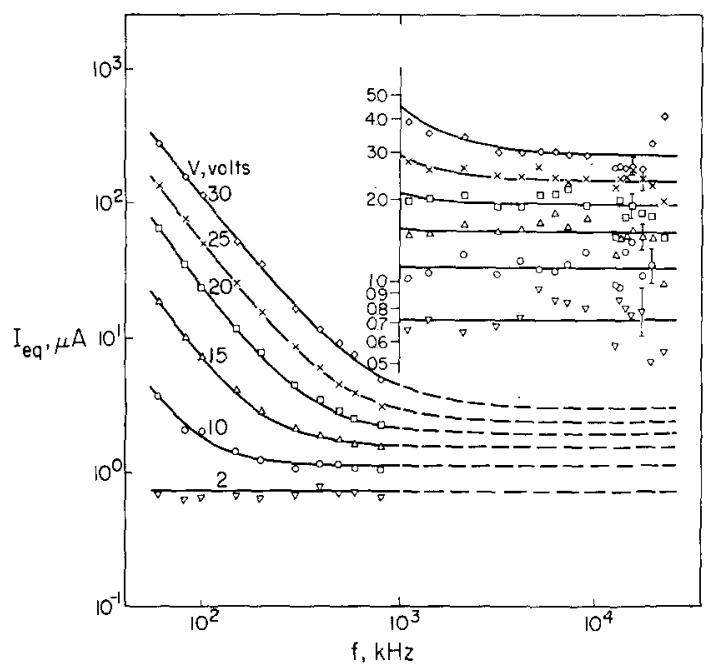

FIG. 7. Noise power spectra at six operating points (see Fig. 3). The solid lines are least-squares fits of $I_{\mathrm{eq}}=c_{1}+c_{2} / f^{c_{3}}$ to the data. The insert displays data and fits above $1 \mathrm{MHz}$.

11 "Nyquist noise" is adopted here to refer specifically to quasithermal noise of quasilinear systems in which $\left\langle i^{2}\right\rangle=4 k T g \Delta f$, where $g$ is a linear element characterizing the (nonlinear) system which is not in true thermal equilibrium. "Thermal noise" is more broadly interpreted to refer to any type of noise due to thermal agitation. 
which play a dominant role in establishing the static characteristic, are too slow to affect the current at frequencies $\omega \gg 1 / \tau_{1}$. This fact is also expressed by the straight line $I=V / r$ in Fig. 1 . We suggest that the highfrequency fluctuations of this current are not affected by the recombination processes either. Rather, we maintain that the noise originates from thermal fluctuations in $r$.

A different explanation starts from Eq. (3) and Fig. 2. The current step $\Delta i(0)$ is determined by the electron and hole concentrations $n$ and $p$ immediately before the voltage step $\Delta V$ is applied. Electrons and holes contribute independently to the flow at that instant and without changing their concentrations. Van der Ziel and van Vliet proved recently that carriers of a single type injected into a volume element of a device will generate thermal noise. ${ }^{12}$ If, in our device, recombination is the only mechanism coupling the holes to the electrons and this mechanism is ineffective at $\omega \gg 1 / \tau_{1}$, van der Ziel's and van Vliet's conclusion applies to both electrons and holes independently. One then must expect

$$
\begin{aligned}
\left\langle i^{2}\right\rangle & =4 k T\left\{\left[A \sigma_{n}\left(\omega \gg 1 / \tau_{1}\right) / L\right]+\left[A \sigma_{p}\left(\omega \gg 1 / \tau_{1}\right) / L\right]\right\} \Delta f \\
& =4 k T g \Delta f, \quad \omega \tau_{1} \gg 1 .
\end{aligned}
$$

It is thus clear that the interpretation of the high frequency noise requires more than the knowledge of the static $I-V$ characteristic. Therefore, recent treatments with the results $\left\langle i^{2}\right\rangle=n 4 k T g \Delta f^{13}$ and $\left\langle i^{2}\right\rangle=$ $\left[4 \mu_{p} \mu_{n} /\left(\mu_{p}+\mu_{n}\right)^{2}\right] 4 k T g \Delta f^{14}$ appear to be incorrect in double injection diodes. Instead the fact should be used that at high frequencies $V=$ const $\cdot I=r I$ which

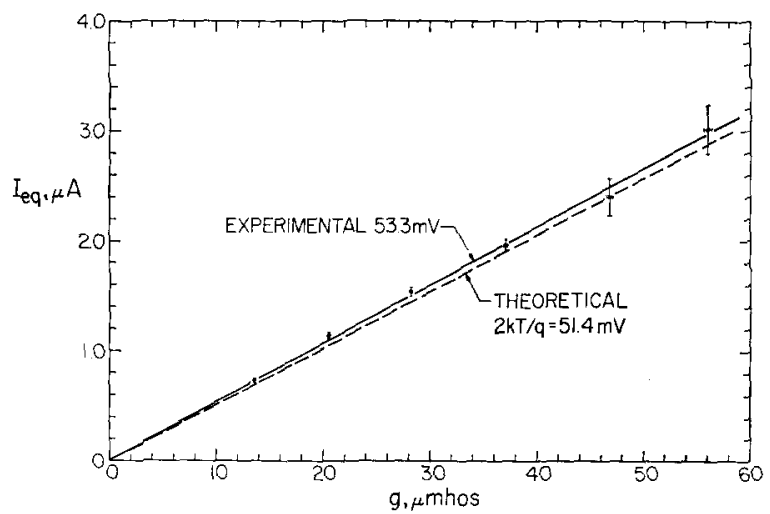

Fĩg. 8. High-frequency equivalent noise current $I_{\mathrm{ea}}$ vs highfrequency conductance $g=1 / r$. The ratio of the slope of the solid line (least-squares fit) to the slope of the dashed theoretical line $(2 k T / q)$ is $\alpha=1.04 \pm 0.05$.

${ }^{12}$ A. van der Ziel and K. M. van Vliet, Solid-State Electron. 11,508 (1968).

${ }^{13}$ S. Yamamoto, S. T. Liu, and A. van der Ziel, Appl. Phys. Letters 11, 140 (1967); see also J. S. Barrera, thesis, Carnegie Institute of 'Technology (1966).

${ }^{14} \mathrm{~A}$. van der Ziel, IEEE Trans. MTT 16, 308, 1968. The correct relation between low-frequency conductance $g_{0}$ and highfrequency conductance $g$ of the Si double-injection diode mentioned in this reference is $g=g_{0} / 2$. It may also be mentioned that the term "noise suppression" of Ref. 1 is not appropriate in double injection as long as it refers to thermal noise hypotheses.

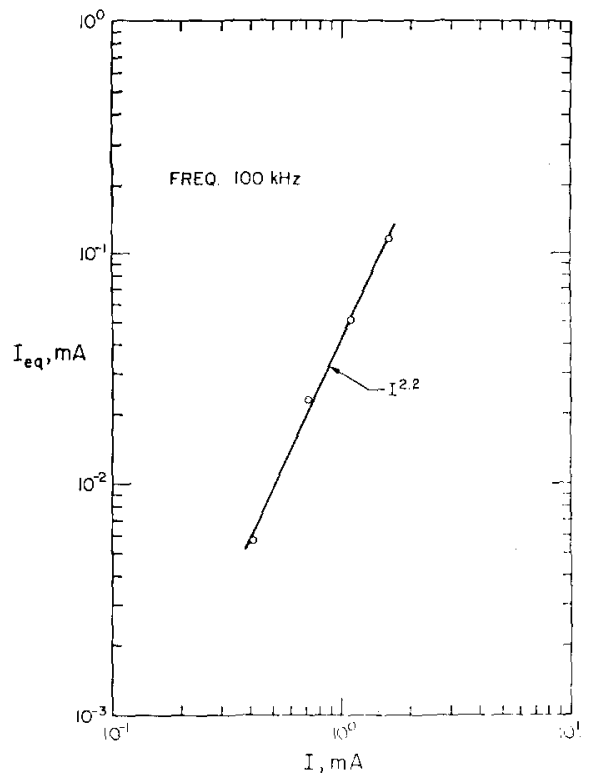

Fig. 9. Observed excess noise at $100 \mathrm{kHz}$ vs diode current at four operating points. The slope is close to $2 . I_{\text {eq }}$ is the equivalent noise current obtained at $100 \mathrm{kHz}$ from the dependence $I_{\text {eq }}=c_{1}+c_{2}^{\prime} / f^{2}$ when least-squares fitted to the experimental points of Fig. 7 .

leads immediately to Eqs. (9) and (8). The thermal noise current source $\left\langle i^{2}\right\rangle=4 k T g \Delta f$ in the equivalent circuit of Fig. 4 expresses these ideas formally.

With the high-frequency noise reduced to thermal causes, the interesting question now arises as to when $\alpha$ ceases to be unity. The fact that $\alpha=1$ for devices as different as those of Refs. 1-3 and the simplicity of the model offered obviously speak for a broad validity of this concept. On the other hand, experiments on single injection devices yield $\alpha=2 .{ }^{13}$ There is, however, an important difference between these two cases. In double injection, the dielectric relaxation time $\theta$ is quite small, $\tau_{1}$ is fairly large, as in the present case, and the transit times $t_{n}$ and $t_{p}$ of electrons and holes are of a magnitude $t_{0}$ similar to that of $\tau_{1}$. Hence, there is a wide frequency range confined by the limits $1 / \tau_{1}<\omega<$ $1 / \theta$. In this range double injection devices are linear elements when diffusion is negligible because recombination is absent at these frequencies. Generally then, $1 / \tau_{1}<\omega$ appears as a necessary condition for thermal high-frequency noise if $\tau_{1}$ represents the shortest lifetime of all recombinations processes at work. This condition is also satisfied formally in single injection, since recombination is absent even under static conditions. In single injection, however, the dielectric relaxation time $\theta$ is of the same order of magnitude as the transit time $t_{0}$, while in double injection $\theta \ll t_{0}$. One might thus speculate that a condition of the form $1 /\left(t_{0}, \tau_{1}\right)<\omega<1 / \theta$ is necessary for the existence of linear behavior of the devices and for Nyquist noise at high frequencies. Another natural time constant of a device is the mean free time $\tau_{c}$ between collisions for the charge carriers. A time of this order is needed to 
"thermalize" the carrier motion. We expect, therefore, noise which is quite different from Nyquist noise at frequencies $\omega_{c} \sim 1 / \tau_{c}$. Unfortunately, $\omega_{c}$ is usually in the microwave range which complicates the experimental situation. The dependence of the Nyquist noise on temperature offers an additional possibility to test the present hypothesis of high-frequency noise. Experiments to determine this temperature dependence are under preparation.

Implied in our hypothesis is the idea that the $\mathrm{dc}$ current of the double-injection diode merely serves the purpose of maintaining a steady-state nonequilibrium distribution of charge carriers. Conceivably this could be achieved also by other means, such as radiation. The high-frequency noise of the device should still be given by Eq (9). Experiments of this nature seem very worthwhile. We also believe that the high-frequency noise of such devices, if properly designed and operated, will offer unique opportunities to study fluctuations of tepid and hot charge carriers in a solid.

\section{SPECULATIONS ABOUT LOW-FREQUENCY NOISE IN DOUBLE INJECTION}

As outlined above, the high-frequency noise does not reveal anything unique to the double-injection process. Turning to lower frequencies we may anticipate generation-recombination $(g-r)$ noise since electrons and holes are present in similar concentrations over most of the volume.

A picture of the $g-r$ noise can be developed along the following lines:

(1) The $g-r$ process has one time constant $\tau(=$ lifetime).

(2) The fluctuations in a volume element are given by $\left\langle\Delta N^{2}\right\rangle=\left\langle\Delta P^{2}\right\rangle=N P /(N+P)$, where $N$ and $P$ are the total numbers of electrons and holes in that volume element. ${ }^{15}$

(3) The noise voltage across the (one-dimensional) device is obtained from the integral

$$
\int_{0}^{L}\left\langle v^{2}\right\rangle d x
$$

where $\left\langle v^{2}\right\rangle$ is the mean square voltage fluctuation across $d x$.

(4) Diffusion is neglected. The result is expressed as $I_{\text {eq, }, \mathrm{b}-\mathrm{r}}$ :

$$
\begin{aligned}
I_{\mathrm{eq}, \mathrm{g}-\mathrm{r}}=\frac{2(1+b)^{2}}{q^{3} \mu_{p}^{2} A^{3}} & \frac{\tau}{\left[1+(\omega \tau)^{2}\right]} I^{2}[g(\omega)]^{2} \\
& \times \int_{0}^{L} \frac{(n / p) d x}{(1+n / p)(1+b n / p)^{4} p^{3}},
\end{aligned}
$$

where $n$ and $p$ are functions of $x$. An estimate of $p(x, I)$ is $^{9}$

$$
p(x, I)=\left\{(\tau \pi I / q A)\left[\mu_{n} \mu_{p} /\left(\mu_{n}+\mu_{p}\right)^{2}\right] 1 / x\right\}^{1 / 2} .
$$

${ }^{15}$ A. van der Ziel, Fluctuation Phenomena in Semiconductors (Butterworths Scientific Publications, London, 1959), Chap. IV.
The dominant contribution to the integral stems from the region $x \sim L$. where $p$ has a minimum of about $\pi$ (residual doping of the bulk) and $n \sim p$ is assumed. In general we would expect

$$
I_{\text {eq, }, \mathrm{r}-\mathrm{r}} \propto I^{n}, \quad \frac{3}{2} \leq n \leq 4 .
$$

An estimate of the magnitude of $I_{\text {eq, } \mathrm{g}-\mathrm{r}}$ at $1.6 \mathrm{~mA}$ and $100 \mathrm{kHz}\left(\omega \tau_{1}=24\right)$ is $30 \mu \mathrm{A}$. This is within an order of magnitude of the measured excess noise shown in Fig. 9. This estimate leads to the two following conclusions: (i) It is probable that $g-r$ noise is the main contribution to the low-frequency noise in double injection. It may be very difficult to identify quasithermal noise sources like those mentioned in Refs. 2 and 3. (ii) It is a worthwhile endeavor to investigate the detailed features of $g-r$ noise in double injection. ${ }^{16,17}$

\section{vi. CONCLUSIONS}

The admittance and step-response measurements on a long silicon double-injection diode at room temperature demonstrate that this device can be represented by the equivalent circuit of Fig. 4 which can be quantitatively derived from existing models of double injection.

Noise measurements on this device at high frequencies show that the white noise at all operating points considered is given by the Nyquist noise of the highfrequency resistor $r$ of the equivalent circuit. This result is explained in Sec. IV without additional hypotheses on the physics of the double injection process. Based on this and the fact that measurements at operating points below the double-injection regime as well as those from Refs. 2 and 3 all result in Nyquist noise, we propose that this result is not restricted to double injection alone. Section IV speculates on the limits of this, we think, rather general phenomenon.

The experimental evidence and first-order calculations on the low-frequency noise suggest a generationrecombination mechanism as the prime source of noise. A closer analysis of this as well as the high-frequency noise under modified conditions of the mode of double injection appear as promising subjects for future investigations.

\section{ACKNOWLEDGMENTS}

Our acknowledgments go to the Naval Ordnance Test Station, Pasadena, California; the Jet Propulsion Laboratory, Pasadena, California; and the NASA Electronics Research Center, Cambridge, Massachusetts, all of whom supported phases of this work. We thank $\mathrm{N}$. Haralambis for much help in the assembly of electronic equipment and data reduction and Dr. R. Baron for enlightening discussions. The double-injection diode was generously provided by $\mathrm{O}$. J. Marsh, Hughes Research Laboratories, Malibu, California

\footnotetext{
${ }^{16}$ This opinion was voiced independently by A. van der Ziel (private communication).

17 A. B. Fazakas and A. Friedman, Phys. Status Solidi 28,
} 385 (1968). 